\title{
Delayed matching-to-successive-samples in pigeons: Short-term memory for item and order information
}

\author{
SUZANNE E. MACDONALD \\ York University, Toronto, Ontario, Canada
}

\begin{abstract}
Short-term memory for order information in pigeons was explored by using a delayed matchingto-successive-samples task (DMTSS). Experiment 1 indicated that pigeons can accurately report the order of two successively presented samples. Experiments 2, 3 and 4 specifically addressed the representation of order information in short-term memory. Experiment 2 showed that when the duration of the first sample (S1) was very long, or when the duration of the second sample (S2) was very short, order errors increased relative to baseline (S1 and S2 of equal duration), suggesting that memory strength plays an important role in the discrimination of order. The possibility that strength information is necessary for accurate DMTSS performance was tested in Experiments 3 and 4. Pigeons continued to match accurately when memory strength and order were uncorrelated.
\end{abstract}

Previous research directed specifically at the processing of sequences of events has shown that animals can learn to discriminate and to produce sequences of temporally ordered stimuli. Sequence discrimination experiments require animals to discriminate a particular sequence of stimuli from several possible alternative sequences. Typically, responding after only one of many possible sequences is reinforced. The results of sequence discrimination studies have shown that animals can accurately discriminate two- and three-element sequences (e.g., Cowey \& Weiskrantz, 1976; D’Amato \& Columbo, 1988; Roitblat, Scopatz, \& Bever, 1987; Terrace, 1987; Weisman \& DiFranco, 1981; Weisman, Duder, \& von Konigslow, 1985; Weisman, Gibson, \& Rochford, 1984; Weisman, Wasserman, Dodd, \& Larew, 1980). Sequence production studies require animals to respond to a set of stimuli in a particular order. The reinforced order remains constant across trials, although the configuration of stimuli varies randomly from trial to trial. A considerable amount of research has shown that pigeons can learn to produce sequences of up to five elements with no explicit intratrial feedback and can generalize this production to novel stimulus configurations (e.g., Straub, Seidenberg, Bever, \& Terrace, 1979; Straub \& Terrace, 1981; Terrace,

This research was part of a doctoral dissertation submitted by the author to the University of Alberta. It was supported in part by a NSERC postgraduate scholarship and by a University of Alberta Dissertation fellowship. Preparation of this article was supported by a NSERC Operating Grant to the author. Thanks to Douglas S. Grant, C. Don Heth, Marcia L. Spetch, Ron Weisman, and especially to Peter Urcuioli, for their valuable comments on previous drafts. Correspondence concerning this article should be addressed to Suzanne E. MacDonald, Department of Psychology, York University (Atkinson College), 4700 Keele St., Toronto, ON, Canada M3J IP3 (e-mail: suzmac@vm1.yorku.ca). 1986a, 1986b, 1987, 1991; Terrace \& Chen, 1991a, $1991 \mathrm{~b})$. In addition, pigeons can perform quite accurately on subsets of the training sequence, indicating that they do not simply learn stimulus-response chains in the original sequence production task.

Taken together, the results of these studies suggest that birds can learn about the relative order of elements in a sequence and may use a representation of this order to perform accurately on these types of tasks. It has been suggested that animals solve discrimination/production problems by comparing a short-term memory representation of the current sequence with a reference or longterm memory representation of the reinforced sequence(s) (e.g., Terrace, 1986a; Weisman, Gibson, \& Rochford, 1984). By this argument, the animal in sequence discrimination studies will respond at the time of test if the current representation matches the reference representation. In production experiments, the animal is assumed to use its reference representation to guide its responses to a set of stimuli. Tasks such as these test long-term representations -in other words, the ability of animals to detect and remember consistent regularities in the order that events are presented. However, environmental contingencies often change, so order is not always constant. Under these circumstances, animals may need to use short-term (or working) memory to update and adapt to these changes. Experiments addressing short-term memory for recent behavior support the notion that animals can retain and utilize order information that varies from trial to trial. For example, numerous studies have shown that pigeons, rats, and monkeys can accurately "report" on their temporally ordered recent behavior (e.g., Grayson \& Wasserman, 1979; Jitsumori \& Sugimoto, 1982; Kramer, 1982; Parker, 1984; Shimp, 1976a, 1976b; Shimp \& Moffit, 1974). 
One method for assessing short-term memory for order information is the delayed matching-to-successive-samples procedure (DMTSS) introduced by Devine and Jones (1975). In DMTSS, memory for sample items and their order of presentation within different sample sequences is tested on every trial. For example, following the successive presentation of two samples, the animal must choose, from among three comparison alternatives, the two stimuli seen previously as the samples and must choose them in the order in which they were presented. For instance, to obtain reinforcement after a sequence consisting of a red sample followed by a green sample, the animal must initially choose the comparison corresponding to the first sample (in this case, red) and then choose the comparison corresponding to the second sample (in this case, green). The third comparison stimulus serves as a distractor, and any responses to it result in trial termination.

The DMTSS paradigm thus requires subjects to discriminate the particular sample sequence presented on each trial and to produce or recall the sequence at the time of testing. Because subjects must remember which samples were presented and the order in which they appeared, this task can be used to assess short-term memory for item and order information. In the experiments reported here, short-term memory for these types of information in pigeons was explored by using a DMTSS task. In Experiment 1 , retention of order information was assessed. Experiments 2, 3, and 4 specifically addressed the representation of order information by manipulating sample duration.

\section{EXPERIMENT 1}

The purpose of this experiment was to determine, by using the DMTSS task developed by Devine and Jones (1975), whether pigeons can retain and recall order information. Accurate performance on this task would indicate that pigeons are capable of retaining information about the order of presentation of two sample stimuli, as well as information regarding their identity.

\section{Method}

\section{Subjects}

Eight experimentally naive Silver King pigeons served as subjects. They were reduced to, and maintained at, $85 \%$ of their free-feeding weight. They were individually housed under 24-h illumination. Water and grit were continuously available in the home cages.

\section{Apparatus}

The birds were tested in eight identical chambers. Three pecking keys were mounted horizontally in a row $20 \mathrm{~cm}$ above the grid floor in each chamber. An Industrial Electronics, Inc. in-line projector was mounted behind each pecking key and projected stimuli onto the pecking keys. The stimuli (red, green, and yellow colored fields) filled the $2.5-\mathrm{cm}$ diameter response key completely. A 28V houselight was mounted directly above the center key, with the housing adjusted so that the emitted light was directed toward the ceiling. The houselight remained illuminated throughout every experimental session, except for brief (3-sec) time-out periods after an incorrect test response. A grain feeder was mounted below the center pecking key, allowing brief (3-sec) access to mixed grain after correct test responses. Each test chamber was enclosed in a sound- and light-attenuating enclosure. Masking noise was provided by an exhaust fan within the enclosure and by white noise delivered through a speaker in the testing room. The presentation of events within the chambers and the recording of data were controlled by microcomputers located in an adjoining room.

\section{Procedure}

Initial training. The birds received magazine training and autoshaping with red, green, and yellow stimuli presented on the center pecking key. After autoshaping, the birds progressed through a series of preliminary training stages. They received 44 sessions of singlesample/two-comparison delayed matching-to-sample (DMTS) training, and then were introduced to single-sample DMTS with three comparison stimuli. After 32 sessions on three-choice matching, performance stabilized at a high level of accuracy (mean accuracy for the final four-session block $=93.2 \%$; range $=85.5$ to 98.0 ). The birds were then introduced to the delayed matching-tosuccessive-samples task. In the initial training version of DMTSS, the birds were required to choose, in any order, the two comparison stimuli (from the three presented) that corresponded to the two sample stimuli on each trial. Mean first-choice accuracy during the final four-session block of this order-irrelevant training task was $81.1 \%$; mean second-choice accuracy was $83.9 \%$. After 36 sessions (2,592 trials) on this order-irrelevant DMTSS task, the birds were introduced to the standard, order-relevant paradigm.

Order-relevant DMTSS. In this version of DMTSS, the birds were required to peck the comparison stimuli in a particular order. Devine and Jones (1975) and Devine, Burke, and Rohack (1979) required their rhesus monkeys to respond in a "forward" order to the comparison stimuli-that is, a response to the comparison corresponding to the first sample ( $\mathrm{S} 1$ ) followed by a response to the comparison corresponding to the second sample (S2) was reinforced. However, pilot testing revealed higher accuracy when the birds were required to respond in a "backward" $\mathbf{S 2 - S 1}$ order. In view of this, and because several researchers have suggested that animals may have an innate tendency to respond to the most recent of a series of stimuli (e.g., Davis \& Fitts, 1976; Roberts and Grant, 1976), the backward order (S2-S1) was designated as correct for the order-relevant task used here. Nevertheless, just as with forward responding, backward responding requires that the animal remember the order of the sample items.

Sessions consisted of 72 trials. Trials were separated by an intertrial interval of $40 \mathrm{sec}$ and began with the presentation of a black dot on a white background as a preparatory stimulus. The preparatory stimulus was presented on the center key for $5 \mathrm{sec}$, or until a response to it occurred. Termination of the preparatory stimulus was followed immediately by a presentation of $S 1$ on the center pecking key. After $5 \mathrm{sec}$, Si was extinguished and followed immediately by a 5 -sec presentation of S2, also on the center key. A short (0.5-sec) delay followed termination of the second sample, after which all three pecking keys were illuminated by the choice stimuli (red, green, and yellow).

A single response to the comparison that corresponded to the sample presented second (S2) extinguished that keylight, leaving a choice between two remaining comparison stimuli. A single response to the comparison that corresponded to the sample presented first (S1) then extinguished the remaining two keys and resulted in 5-sec access to grain. For example, if the sample sequence on a particular trial was red followed by green, reinforcement was delivered only if the bird pecked the green comparison first and then pecked the red comparison. An incorrect comparison response at any time (first or second choice) caused the pecking keys to be extinguished and resulted in both a 5-sec time-out, during which the houselight was turned off, and the termination of that trial. 
Each of the six possible sample-sequence combinations $(R-G$; R-Y; G-R; G-Y; Y-R; Y-G) was presented randomly on 12 trials in each session. The position of the colored comparisons was also randomized with the constraint that each of the six possible comparison-stimulus configurations ( $R-Y-G ; R-G-Y ; G-R-Y$; G-Y-R; Y-R-G; Y-G-R) was presented twice after each sample sequence in each session. The chance probability of a correct first choice in this task was $1 / 3$, or .33 . If, and only if, the bird responded correctly at the time of its first choice did the opportunity to make a second choice arise. The chance probability of a correct second choice was therefore $1 / 2$, or .50 .

After 24 sessions of order-relevant DMTSS, second-choice accuracy had not risen above chance for 4 of the birds. First-choice accuracy, however, was significantly above chance for all birds. Low second-choice accuracy may have been a function of the DMTSS task. Because first-choice responses to $\mathbf{S} 1$ resulted in an immediate time-out (i.e., they were punished), this may have produced the low levels of correct responding to $S 1$ on second choices. To help counteract this potential problem, a "correction" procedure was implemented after the initial 24 sessions. In this procedure, responses to the distractor stimulus on second choices were recorded as distractor errors, but had no programmed effect. Instead, the bird was simply required to move away from the distractor stimulus and respond to the comparison corresponding to $S 1$ before receiving reinforcement. In this way, the bird was forced to respond to $S 1$ on every second choice in order to receive reinforcement, even though incorrect responses continued to be recorded as errors. The correction procedure remained in effect for the duration of Experiment 1 and also in the following experiments. Training with the correction procedure continued for 68 sessions $(4,896$ trials), for a combined total (noncorrection plus correction sessions) of 92 sessions $(6,624$ trials $)$.

\section{Results and Discussion}

\section{Accuracy Data}

Two birds (336 and 338) performed at a chance level of accuracy on both first and second choices; a 3rd bird (332) performed at a chance level on second choices. Because these 3 birds failed to acquire the order-relevant task after 68 sessions, they were dropped from the present series of experiments, and their data are not included in the analyses reported below.

Each of the remaining 5 birds performed at a level significantly above chance on both first and second choices (chi-square tests, all $p s<.05$ ). The mean percentage of

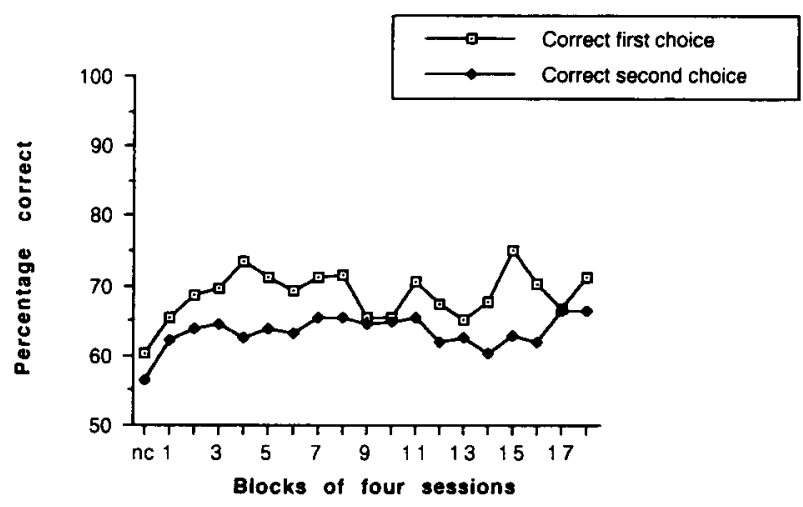

Figure 1. Mean percentage of correct first and second choices during delayed matching-to-successive-samples task in Experiment 1 (nc = final block of noncorrection training). their correct first and second choices is shown in Figure 1. Mean first-choice accuracy during the final four-session block was $71.0 \%$; mean accuracy on second choices was $66.2 \%$. Both of these levels are significantly higher than chance [first choice, $t(4)=12.67, \mathrm{p}<.001$; second choice, $t(4)=6.16, p<.01]$.

\section{Error Data}

There are two types of errors that can occur in the DMTSS task-distractor errors and order errors. Distractor errors occurred when a bird pecked a comparison that did not match either of the two samples. The chance probability of a first-choice distractor error (D1) in this task was $1 / 3$, or .33 ; the chance probability of a second-choice distractor error (D2) in this task was $1 / 2$, or .50. The mean percentage of $\mathrm{Dl}$ errors during the final four-session block was 10.7 (range $=8.3$ to 17.7 ; standard deviation $=$ 4.02), which is significantly lower than that expected by chance $[t(4)=12.63, p<.001)]$. The mean percentage of D2 errors during the final four-session block was 33.8. The percentage of $\mathrm{D} 2$ errors is, of course, equal to [1 -(percentage of correct second choices)], so reporting significance for these types of errors is redundant with the second-choice accuracy data.

Order errors occurred when a bird's initial choice was the comparison corresponding to the first sample item (e.g., pecking the red comparison first after a red-green sample sequence). Assuming equiprobable responding to each of the three comparisons, the chance probability of an order error is .33. During the final four-session block of data collection, the mean percentage of order errors was $18.2 \%$ (range $=14.2$ to 22.2 ; standard deviation $=$ 3.08). The percentage of order errors was significantly lower than that expected by chance $[t(4)=10.94$, $p<.001]$.

The results of this experiment demonstrate that at least some pigeons can retain item and order information in the DMTSS task. First- and second-choice accuracy were consistently above chance for most of the 8 birds. Nonetheless, the DMTSS task is difficult for pigeons, as evidenced by the lack of acquisition shown by 3 of the 8 birds. Other animals also have difficulty mastering this task. Devine, Burke, and Rohack's (1979) rhesus monkeys each required approximately 10,000 trials to reach the criterion level of accuracy, and that task was made easier for 1 monkey by the omission of sample sequences containing two samples from the same dimension (i.e., two colors or two shapes). In the present task, the same three stimuli (all colors) were used as both samples and comparison stimuli throughout training and testing. This probably resulted in the build-up of considerable interference as trials progressed and may have made the task more difficult.

\section{EXPERIMENT 2}

Experiment 2 assessed the effect of changes in samplestimulus duration on the retention of order information in DMTSS. The duration of S1 (with S2 duration held 
constant) and the duration of S2 (with S1 duration held constant) were manipulated. The purpose of this experiment, and of those that follow, was to determine how order information may be represented in short-term memory. Predictions from two major hypotheses about the retention of temporal-order information were tested, as described below.

One method of determining the relative recency of events is to "tag" memories according to their time of presentation (e.g., Yntema \& Trask, 1963). On the basis of this view, temporal attributes are appended to representations of events as the events occur, and recency judgments depend on accessing the temporal tags attached to each representation. Under the time-tag hypothesis, sample duration manipulations should primarily affect the extent to which the identity of sample items is encoded. Increasing sample duration should thus result in a decrease in distractor errors relative to baseline levels. For example, if $\mathbf{S 1}$ duration is increased, a decrease in D2 errors should be observed; if $\mathbf{S} 2$ duration is increased, a decrease in D1 errors should be observed. Decreasing sample duration should produce the opposite effects. Sample duration manipulations may also affect the number of order errors observed, because accurate time tags may depend on accurate sample encoding. In other words, a sample must be encoded in order to be tagged. Under the timetag view, if S1 or S2 durations are very short (thus reducing sample encoding time), an increase in order errors may result.

Another way that order may be represented in memory is through a comparison of relative memory strengths (e.g., Hinrichs, 1970). At the time of testing, S2 should be the stronger memory and S1 the weaker memory, assuming that each item had the same amount of time for encoding when it appeared. The bird may use this memory-strength difference as an order cue to know to which stimulus to respond at testing - that is, in this task "respond to stronger memory first (S2), followed by the weaker memory (S1)." Assuming that sample duration is positively correlated with memory strength, sample duration changes should have a significant effect on accuracy and on the types of errors pigeons make in the DMTSS task. For example, as S1 duration is increased relative to baseline, the memory-strength view predicts more order errors because the relative strength of $\mathrm{S} 1$ would be increased. In other words, the bird should (erroneously) respond to the comparison corresponding to S1 on its first choice more often than on baseline trials. Decreasing the duration of $S 2$ should produce the same effect, because this would decrease the strength of the S2 memory representation. Consequently, the relatively strong S1 representation vis-à-vis $\mathbf{S} 2$ should again produce more first-choice responses to the $S 1$ comparison (i.e., an increase in the number of order errors).

\section{METHOD}

\section{Subjects and Apparatus}

The 5 birds that matched at an above-chance level from Experiment 1 (Birds 331, 333, 334, 335, and 337) served as subjects. The apparatus was the same as that used in the previous experiment.

\section{Procedure}

Sessions consisted of 72 trials. Of these, 36 trials were $S 1$ probe trials, and 36 were $S 2$ probe trials. On $S 1$ probe trials, the duration of $S 1$ was manipulated, with $S 2$ held constant at the baseline duration of $5 \mathrm{sec}$. On S2 probe trials, the duration of S2 was manipulated, with $S 1$ held constant at the baseline duration of $5 \mathrm{sec}$. The range of sample durations tested on both $S 1$ and $S 2$ probes was $1,2,5$, and $8 \mathrm{sec}$, resulting in a total of eight different probe trial types. Each probe trial type was presented for 9 trials during each session. A total of 18 baseline-duration ( $\mathrm{S} 1=5 \mathrm{sec}: \mathrm{S} 2=5 \mathrm{sec}$ ) trials were also presented during each session. To equate the numbers of baseline- and novel-duration trials in the data analyses, 9 of the 18 baseline-duration trials were randomly selected for comparison with the novel-duration probes. The order of trial presentation of the baseline and probe trials and the configuration of the comparison stimuli were randomized within each session. Reinforcement continued to be provided for all correct response sequences. Testing continued for 12 sessions, which provided 108 observations at each probe-type duration.

\section{Results and Discussion}

The mean percentages of correct first and second choices, distractor errors, and order errors at each sample duration (collapsed over 12 sessions) are shown in Figure 2. As is evident from the figure, the sample duration manipulation primarily affected first-choice accuracy and the number of order errors. When S1 duration was very long $(8 \mathrm{sec})$, or when $\mathrm{S} 2$ duration was very short (1 or $2 \mathrm{sec}$ ), many more order errors were made. The increase in order errors was accompanied by a decrease in firstchoice accuracy, especially when $\mathbf{S} 2$ duration was short (1 or $2 \mathrm{sec}$ ). A secondary finding was an increase in firstchoice distractor (D1) errors when either of the two samples was very short $(1 \mathrm{sec})$.

Separate two-factor (blocks and sample duration) analyses of variance (ANOVAs) were initially performed on the data for first-choice accuracy, second-choice accuracy, order errors, and distractor errors. The main effect of blocks and the blocks $\times$ sample duration interaction were not significant in any of the analyses, indicating that the data were highly stable across the 12 testing sessions. The data were thus collapsed across sessions for further analysis.

The main effect of sample duration was highly significant for the first-choice accuracy data $[F(6,24)=9.83$, $p<.001]$. Planned contrasts showed that first-choice accuracy, compared with baseline accuracy, decreased significantly when $S 1=8 \sec [F(1,4)=9.0, p<.04]$, when $\mathrm{S} 2=1 \sec [F(1,4)=27.5, p<.006]$, and when $\mathrm{S} 2=2 \sec [F(1,4)=35.2, p<.004]$.

The main effect of sample duration was also significant for order error data $[F(6,24)=11.17, p<.001]$. Planned contrasts showed that order errors increased significantly relative to baseline when $S 1=8 \sec [F(1,4)=$ $36.58, p<.004]$, when $S 2=1 \sec [F(1,4)=18.47$, $p<.01]$, and when $\mathrm{S} 2=2 \sec [F(1,4)=28.19$, $p<.006]$.

The primary finding of Experiment 2 was an increase in order errors when S1 duration was very long, or when S2 duration was very short, relative to baseline duration. This result strongly supports the memory-strength hypothesis of order representation. According to this view, S1 

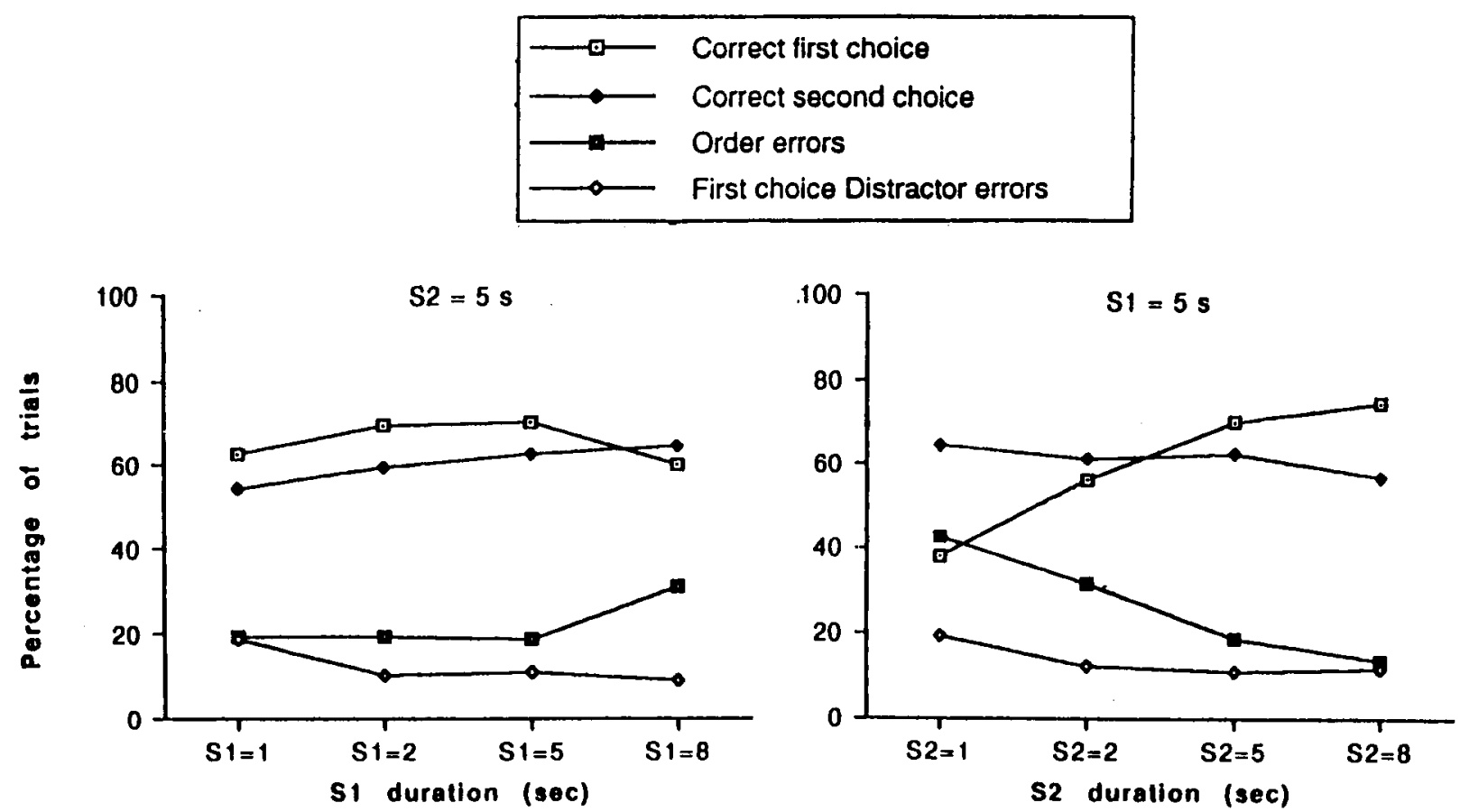

Figure 2. Mean percentage of correct first and second choices and errors at each sample duration in Experiment 2. Note: The datum point for $S 1=5: S 2=5$ represents the baseline level of responding. It is plotted in both the S1 and the S2 functions.

and $\mathrm{S} 2$ establish separate representations in memory. The strength of each representation increases as the duration of sample exposure increases, and decreases as a function of time or intervening trial events. In the baseline condition of the current DMTSS task, S1 and S2 were each presented for $5 \mathrm{sec}$, so presumably the strengths of the individual representations were comparable when each sample was terminated. However, because $S 1$ ended before the presentation of $S 2$, the relative strength of $S 1$ should have been considerably weaker at the time of testing. Consequently, if the pigeons responded on the basis of the relative strengths of $S 1$ and $S 2$, they would have responded first to the comparison corresponding to the (stronger) S2 representation and then to the comparison corresponding to the (weaker) S1 representation.

By this account, increasing the duration of $S 1$ to $8 \mathrm{sec}$ increased the relative strength of $\mathrm{S} 1$, thus producing a significant rise in the number of order errors. Similarly, decreasing S2 duration to 1 or $2 \mathrm{sec}$ also produced significantly more order errors by having the same net effect (i.e., a relatively stronger $S 1$ representation). In other words, the pigeons tended to report that S1 occurred more recently than $\mathrm{S} 2$ because, at the time of test, the difference in strength between the representations of $S 1$ and $\mathrm{S} 2$ was reduced relative to that on baseline trials. If pigeons use the strength difference as an order cue in the DMTSS task, reducing this discrepancy should diminish first-choice accuracy by increasing order errors, similar to what was observed in Experiment 2.

\section{EXPERIMENT 3}

Although the results of Experiment 2 are consistent with a memory-strength hypothesis of order representation, the results are also consistent with a view that maintains that order information is not directly relevant in the DMTSS task. According to this view, pigeons simply discriminate between the relative strengths of the two sample representations and respond on that basis. Although the present DMTSS task is nominally an order-relevant task, it may be functionally an order-irrelevant task. It is possible that the pigeons perceive the task simply as a discrimination between strong and weak memory representations. Under the baseline reinforcement contingencies, a first-choice response to the comparison corresponding to the stronger representation (S2), followed by a second-choice response to the comparison corresponding to the weaker representation (S1), will result in reinforcement. According to this view, the actual order of presentation of the two samples is not directly relevant to successful DMTSS performance. This possibility was explored in Experiment 3.

When the durations of $S 1$ and $S 2$ are equal, as in baseline training sessions (each sample was presented for $5 \mathrm{sec}$ ), order information and relative memory strengths are confounded. That is, the two samples are not only temporally ordered (S1-S2), but they may be ordered with respect to memory strength as well (weak-strong). In Experiment 3 , the pigeons received training sessions in which $\mathbf{S 1}$ duration was $8 \mathrm{sec}$ and $\mathbf{2} 2$ duration was $1 \mathrm{sec}$. 
On the assumption that memory strength is directly related to sample duration, this training situation should result in a relatively strong $S 1$ representation and a relatively weak $S 2$ representation. The memory-strength and order-relevant hypotheses make different predictions regarding the outcome of this manipulation.

According to the memory-strength view, the duration manipulation (8-sec S1:1-sec S2) should result in a drastic reduction in first-choice accuracy. Specifically, most first-choice responses will be to the comparison corresponding to the stronger memory representation (S1), and so order errors will occur on most trials.

The alternative hypothesis states that pigeons view the DMTSS procedure as order-relevant and use relative memory strengths as a cue to the temporal order of the two samples. The results of Experiment 2 strongly suggest that pigeons respond to the comparison corresponding to the stronger representation on first choices, followed by second-choice responses to the weaker representation. Upon introduction of the 8:1 condition, then, most firstchoice responding should be to the comparison corresponding to the stronger representation (S1), and thus a preponderance of order errors should occur. However, if pigeons can attend to temporal order information independently of memory strength, first-choice accuracy should increase as training trials progress.

\section{Method \\ Subjects and Apparatus \\ The subjects and apparatus were the same as in Experiment 2.}

\section{Procedure}

Sessions consisted of 72 trials. The trials were identical to the training trials of the previous experiments, except that the duration of $S 1$ was $8 \mathrm{sec}$ and the duration of S2 was $1 \mathrm{sec}$. The order of presentation of the trials and the configuration of comparison stimuli were randomized within each session.

\section{Results and Discussion}

After the introduction of the 8-sec (S1):1-sec (S2) trials, mean first-choice accuracy dropped to a near-chance level (mean for first block $=37.1 \%$ ). The drop in first-choice accuracy was primarily due to a significant increase in order errors (mean $=41.5 \%$ ) relative to the final block of baseline sessions that immediately preceded Experiment 3 (mean $=19.7 \%)[t(8)=6.53, p<.001]$. Second-choice accuracy and the number of D1 errors did not differ from those on the immediately preceding baseline sessions ( $t$ tests, both $p s>.05$ ).

First-choice accuracy for two of the five birds ( 334 and 335) was significantly above chance in the first block of four sessions (individual chi-square tests, both $p s<.05$ ). Mean acquisition data are shown in Figure 3. As the figure illustrates, mean first-choice accuracy gradually increased across blocks of training. All 5 birds eventually matched at a level above chance (individual chi-square tests, all $p s<.05$ ). However, 1 bird (333) only began matching at above-chance levels after 56 sessions, so its data were not included in Figure 3 or in further data anal-

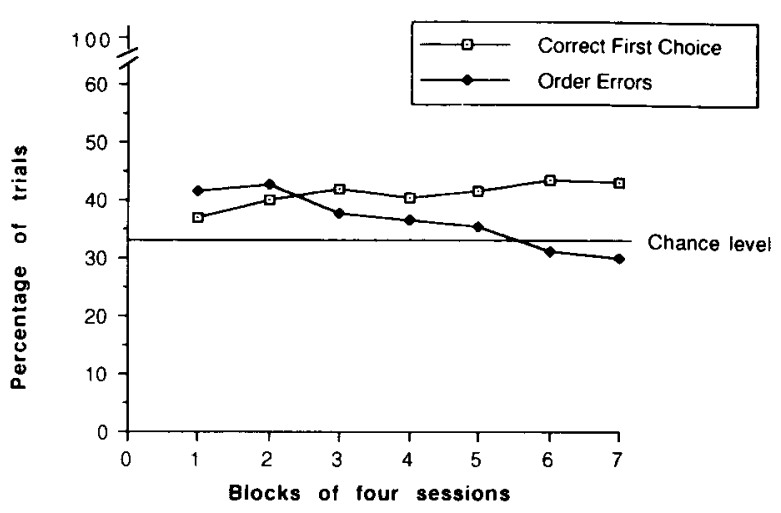

Figure 3. Mean percentage of correct first choices and order errors on 8 sec: 1 sec trials in Experiment 3.

yses. Analyses of the data for the remaining birds were conducted only over the first seven blocks of testing, because some birds had completed testing by that time.

The steady increase in mean first-choice accuracy for 4 of the 5 pigeons is evidenced by a significant positive linear trend across blocks of testing $[F(1,18)=15.93$, $p<.001]$. The increase in first-choice accuracy was accompanied by a steady decrease in the mean number of order errors, as evidenced by a significant negative linear trend across blocks of testing $[F(1,18)=9.76, p<.01]$. Mean second-choice accuracy and distractor errors did not vary significantly over blocks of training ( $F$ tests, both ps $>.15$ ).

The results of this experiment show that pigeons can acquire the DMTSS task when the duration of $\$ 1$ is much longer than the duration of S2. The first- and secondchoice accuracy of all 5 birds eventually reached a level above chance, even though they had previously received over 10,000 trials with samples of equal duration. Indeed, 3 of the 5 birds exhibited above-chance levels of firstand second-choice responding by the 8th day of training (Block 2 in Figure 3). This finding suggests that pigeons may utilize temporal order information in this task. However, although suggestive, the results of the present experiment do not rule out the possibility that the observed accurate performance was again based on a comparison of relative memory strengths, because a S1-S2 strength difference very likely did remain in the present experiment. Reinforcement could be obtained if a bird now responded to the comparison corresponding to the weaker representation (S2) on its first choice, followed by a response to the comparison corresponding to the stronger representation (S1) on its second choice. Thus, over the course of training in Experiment 3 the birds could have learned to "reverse" the response rule described earlier. Specifically, in Experiments 1 and 2, the response rule may have been "respond to strong, then respond to weak," and in Experiment 3, the response rule may have been "respond to weak, then respond to strong." The possibility that pigeons were able to formulate a new deci- 
sion rule based on changing task requirements implies considerable flexibility in pigeon memory processing, but it does not necessarily imply that order information was encoded in the present task. This issue was further explored in Experiment 4.

\section{EXPERIMENT 4}

The results of Experiment 3 suggest that pigeons may respond on the basis of order information in the DMTSS task. However, the results do not definitively rule out the possibility that pigeons respond on the basis of relative memory strengths, because relative memory strength and temporal order were still confounded. In this experiment the confound between "memory strength" and temporal order was further reduced. This was accomplished by presenting DMTSS trials in which S1 was equally often the stronger or weaker of the two sample representations. As in Experiment 3, strength was operationalized by manipulating sample duration. Specifically, sample duration was randomly determined on each trial during each session. On half of the trials, $S 1$ duration was $8 \mathrm{sec}$ and S2 duration was $1 \mathrm{sec}$, as in Experiment 3. On the remaining trials, S1 duration was $3 \mathrm{sec}$ and $S 2$ duration was $5 \mathrm{sec}$. Accurate DMTSS performance under these conditions makes it less likely that pigeons respond solely on the basis of memory strengths, because duration (and thus memory strength) is no longer a consistent predictor of reinforcement. Accurate matching in this case would provide strong evidence that pigeons can utilize temporal order information in the order-relevant DMTSS task.

\section{Method}

\section{Subjects and Apparatus}

Four of the 5 birds from Experiment 3 served as subjects. Bird 333 did not participate in Experiment 4 because of time constraints. The apparatus was the same as in the previous experiments.

\section{Procedure}

Sessions consisted of 72 trials that were identical to those of the previous experiments except that sample duration was manipulated. On 36 trials, S1 duration was $8 \mathrm{sec}$ and $\mathrm{S} 2$ duration was $1 \mathrm{sec}$. On the remaining 36 trials, $S 1$ duration was $3 \mathrm{sec}$ and $S 2$ duration was $5 \mathrm{sec}$. The order of presentation of the trials and the configuration of comparison stimuli were randomized within each session.

\section{Results and Discussion}

Mean data from both types of trials are shown in Figure 4. As is evident from the figure, the introduction of 3-sec (S1):5-sec (S2) trials did not significantly disrupt DMTSS accuracy. First-choice accuracy remained at an above-chance level for each bird on both 8-sec:1-sec trials and 3-sec:5-sec trials (individual chi-square tests, all ps $<.05$ ), and did not drop across blocks of testing. As in Experiment 3, the predominant error on both trial types was order errors.

Because the length of testing varied between individuals, data analyses were based only on the first four blocks of testing for each bird. Separate two-factor ANOVAs

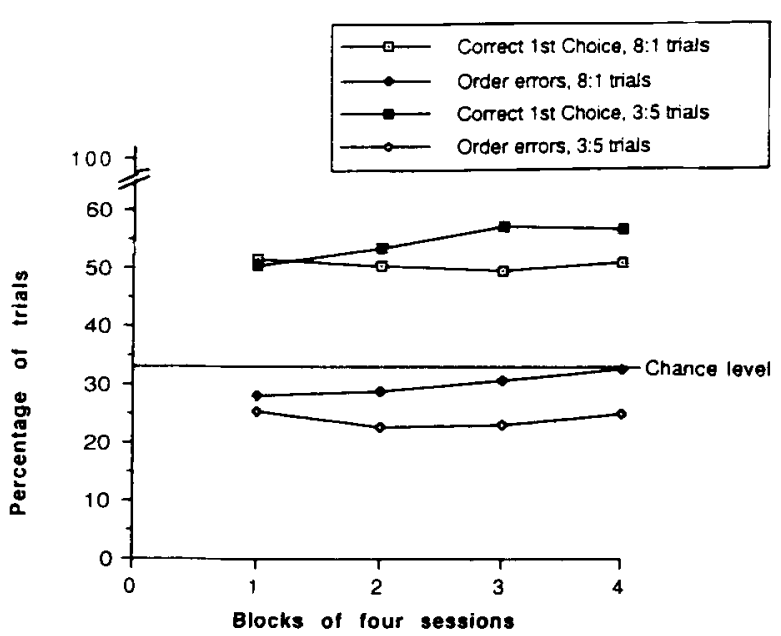

Figure 4. Mean percentage of correct first choices and order errors on 8 sec:1 sec and 3 sec:5 sec trials in Experiment 4.

with blocks of sessions and trial type (8:1 vs. $3: 5$ ) as factors revealed no significant differences in first-choice accuracy, second-choice accuracy, number of order, or D1 errors between the two types of trials.

The results of this experiment show that pigeons can match accurately on the DMTSS task when the relative duration of $S 1$ and $S 2$ varies across trials. This is important, because it makes it less plausible to argue that pigeons use only relative memory strength differences to perform accurately. It may be that pigeons in this situation use a complex response rule such as "if S1 was longer than S2, respond to the comparison corresponding to the weaker memory first; if S1 was shorter than S2, respond to the comparison corresponding to the stronger memory first," but such a possibility seems unlikely. If pigeons do not rely on memory strength information to accurately reproduce the order of two successively presented stimuli in the randomized duration task, what cues do they rely on? A definitive answer awaits further research with this paradigm, but the results certainly suggest that pigeons may rely on the order of the two samples, independent of memory strength.

\section{GENERAL DISCUSSION}

A DMTSS task was used in the series of experiments reported here to address the short-term retention of order information in pigeons. The results of Experiment $1 \mathrm{dem}-$ onstrated that pigeons could retain and recall order information when a unique sample sequence was presented on each trial. Experiments 2, 3, and 4 explored the representation of order information in the DMTSS task. Experiment 2 involved a manipulation of sample duration. The primary finding from this experiment was that pigeons made more order errors when either S1 duration was long or $\mathbf{S} 2$ duration was short (relative to their baseline values), which supports a memory-strength hypothesis of order 
representation. According to this hypothesis, in the standard DMTSS task (S1 and S2 presented for equal durations), pigeons reproduce the order of presentation of the two sample items by comparing the relative memory strengths of the items at the time of test. In Experiments 3 and 4, a more extreme hypothesis was tested-namely, that pigeons can perform accurately on the DMTSS task only when a strength differential exists between the two sample stimuli. According to this hypothesis, the order of the two sample stimuli does not figure directly in accurate DMTSS performance. Rather, accurate matching is the result of a discrimination between strong and weak memory representations. The results of Experiment 4 demonstrated that the pigeons matched accurately when the strength/order confound was reduced by randomizing sample duration lengths. Memory strength may be important in the standard (equal sample duration) DMTSS task, but when task demands change, birds can continue to match accurately when strength is not a consistent predictor of reinforcement.

These results may be best accommodated by postulating the existence of a decision process that operates in pigeons' short-term memory. The decision process is assumed to be the application of a "rule," or instruction, stored in long-term (or reference) memory. This rule is acquired over training and is dependent on the reinforcement contingencies present in the particular task. In this sense, the decision process may be viewed as a controlled process similar to the process of maintenance rehearsal (e.g., Grant, 1984; Maki, 1981) because it is not an invariant characteristic of the memory system. In addition, not all birds may acquire the decision rule, given that 3 of the original 8 birds in this study never performed at above chance levels on the DMTSS task.

According to the memory-strength hypothesis, pigeons determine the order of the two successively presented samples by comparing the relative strengths of the sample representations. In this case, the reference decision rule may be "respond to the comparison corresponding to the stronger (S2) representation first, and then respond to the comparison corresponding to the weaker (S1) representation." According to this view, order information as such is not encoded but is derived from item information. This does not imply that order information is never encoded by pigeons. For example, the results of sequence production and discrimination studies strongly suggest that pigeons can and do encode temporal order information. Similarly, in Experiment 4 of the present series, the birds continued to match accurately in the DMTSS task when the order of the two sample stimuli was the only consistent cue available to guide performance. Further research with randomized sample durations is necessary to determine how order information may be encoded in that paradigm. In the standard DMTSS task, however, it appears that order information is secondary to item information. Pigeons must encode and retain two different sample items on every trial in DMTSS, and the addition of order information may tax the cognitive capacities of these animals. It may be that application of a decision rule based on relative memory strengths is the most efficient way to deal with this difficult task.

\section{Implications}

The finding that memory representation strength may be involved in the DMTSS task has important implications for the study of short-term memory. Several researchers have suggested that short-term, or working, memory is primarily concerned with the recency of events. Staddon (1984) defined working memory as event memory, which is "memory for how long ago something happened, as opposed to its significance in terms of other events" (p. 322). Similarly, D'Amato (e.g., 1973) suggested that the delayed matching-to-sample paradigm can be conceptualized as a temporal discrimination task. According to this view, accurate DMTS performance depends on the animal's ability to discriminate the most recently presented sample stimulus at the time of test. One method of determining the relative recency of events is to tag memories based on their time of presentation (e.g., Yntema \& Trask, 1963). Accordingly, temporal attributes are appended to representations of events, and recency judgments depend on accessing the temporal tags attached to each representation. However, the results of the present series of experiments suggest that judgments of recency in some cases may be based on the relative strengths of memory representations, rather than on time tags. Data from Experiment 2 are particularly instructive in this regard. If the representations of $S 1$ and $S 2$ are tagged according to their order of presentation, manipulation of sample duration should not affect DMTSS accuracy. However, increasing S1 duration and decreasing S2 duration tended to reverse the order of responding in the DMTSS task. This was most pronounced when S2 was at its shortest value. It is difficult to explain this result in terms of time tags, but it is easily accommodated within a strength conception.

Memory strength models have also received some support from studies in which human subjects were used (Hinrichs, 1970; Wickelgren, 1972; Wickelgren \& Norman, 1966). These models have for the most part been replaced by conceptions of temporal coding involving contextsensitive associations (e.g., Toglia \& Kimble, 1976; Tzeng, Lee, \& Wetzel, 1979; Zacks, Hasher, Alba, Sanft, \& Rose, 1984). However, Tzeng et al. (1979) allowed that in some situations, people may judge the relative recency of items on the basis of memory strengths. Specifically, humans may rely on memory strength cues when the number of contextual cues available is minimized. Interestingly, most studies that involve an investigation of animal memory explicitly seek to minimize contextual cues. It may be that under similar circumstances both animals and humans rely on the relative strengths of memory representations to judge the temporal order of events.

\section{REFERENCES}

Cowey, A., \& Weiskrantz, L. (1976). Auditory sequence discrimination in Macaca mulatta: The role of the superior temporal cortex. Neuropsychologia, 14, 1-10. 
D'AMato, M. R. (1973). Delayed matching and short-term memory in monkeys. In G. H. Bower (Ed.), The psychology of learning and motivation: Advances in Research and Theory (Vol. 7, pp. 227-269). New York: Academic Press.

D'Amato, M. R., Columbo, M. (1988). Representation of serial order in monkeys (Cebus apella). Journal of Experimental Psychology: Animal Behavior Processes, 14, 131-139.

Davis, R. T., \& FitTs, S. S. (1976). Memory and coding processes in discrimination learning. In D. L. Medin, W. A. Roberts, \& R. T. Davis (Eds.), Processes of Animal Memory (pp. 167-180). Hillsdale. NJ: Erlbaum.

Devine, J. D., Burke, M. W., \& Rohack, J. J. (1979). Stimulus similarity and order as factors in visual short-term memory in nonhuman primates. Journal of Experimental Psychology: Animal Behavior Processes, 5, 335-354.

DEvine, I. D., JoNes, L. C. (1975). Matching to successive samples: A multiple-unit memory task with rhesus monkeys. Behavior Research Methods \& Instrumentation, 7, 438-440.

GraNt, D. S. (1984). Rehearsal in pigeon short-term memory. In H. L. Roitblat, T. G. Bever, \& H. S. Terrace (Eds.), Animal cognition (pp. 99-116). Hillsdale, NJ: Erlbaum.

Grayson, R. J., \& Wasserman, E. A. (1979). Conditioning of tworesponse patterns of key pecking in pigeons. Journal of the Experimental Analysis of Behavior, 31, 23-29.

HinRICHs, J. V. (1970). A two-process memory-strength theory for judgment of recency. Psychological Review, 77, 223-233.

Jitsumori, M., \& Sugimoto, S. (1982). Memory for two stimulusresponse items in pigeons. Journal of the Experimental Analysis of Behavior, 38, 63-70.

Kramer, S. P. (1982). Memory for recent behavior in the pigeon. Journal of the Experimental Analysis of Behavior, 38, 71-85.

MAKI, W. S. (1981). Directed forgetting in animals. In N. E. Spear \& R. R. Miller (Eds.), Information processing in animals: Memory mechanisms. Hillsdale, NJ: Erlbaum.

PARKER, D. K. (1984). Reproduction memory of two-event sequences in pigeons. Journal of the Experimental Analysis of Behavior, 41, 125-141.

Roberts, W. A., Grant, D. S. (1976). Studies in short-term memory in the pigeon using the delayed matching-to-sample procedure. In D. L. Medin, W. A. Roberts, \& R. T. Davis (Eds.), Processes of animal memory. Hillsdale, NJ: Erlbaum.

Roitblat, H. L., Scopatz, R. A., \& Bever, T. G. (1987). The hierarchical representation of three-item sequences. Animal Learning \& Behavior, 15, 179-192.

Shimp, C. P. (1976a). Short-term memory in the pigeon: Relative recency. Journal of the Experimental Analysis of Behavior, 25, 55-61.

Shimp, C. P. (1976b). Short-term memory in the pigeon: The previously reinforced response. Journal of the Experimental Analysis of Behavior, 26, 487-493.

Shimp, C. P., Mofrit, M. (1974). Short-term memory in the pigeon: Stimulus-response associations. Joumal of the Experimental Analysis of Behavior, 22, 507-512.

Staddon, J. E. (1984). Time and memory. Annals of the New York Academy of Sciences, 423, 322-324.

Straub, R. O., Seidenberg, M. S., Bever, T. G., \& Terrace, H. S.
(1979). Serial leaming in the pigeon. Joumal of the Experimental Analysis of Behavior, 32, 137-148.

Straub, R. O., \& Terrace, H. S. (1981). Generalization of serial learning in the pigeon. Animal Learning \& Behavior, 9, 454-468.

TerRace, H. S. (1986a). A nonverbal organism's knowledge of ordinal position in a serial learning task. Journal of Experimental Psychology: Animal Behavior Processes, 12, 203-214.

Terrace, H. S. (1986b). Positive transfer from sequence production to sequence discrimination in a nonverbal organism. Journal of Experimental Psychology: Animal Behavior Processes, 12, $215-234$.

Terrace, H. S. (1987). Chunking by a pigeon in a serial learning task. Nature, 325, 149-151

Terrace, H. S. (1991). Chunking during serial leaming by a pigeon: I. Basic evidence. Journal of Experimental Psychology: Animal Behavior Processes, 17, 81-93.

Terrace, H. S., Chen, S. (1991a). Chunking during serial learning by a pigeon: II. Integrity of a chunk on a new list. Journal of Experimental Psychology: Animal Behavior Processes, 17, 94-106.

Terrace, H. S., Chen, S. (1991b). Chunking during serial learning by a pigeon: III. What are the necessary conditions for establishing a chunk? Joumal of Experimental Psychology: Animal Behavior Processes, 17, 107-118.

TogliA, M. P., \& Kimble, G. A. (1976). Recall and use of serial position information. Journal of Experimental Psychology: Human Learning and Memory, 2, 431-445.

TzenG, O. J. L., LeE, A. T., \& Wetzel, C. D. (1979). Temporal coding in verbal information processing. Journal of Experimental Psychology: Human Learning \& Memory, 5, 52-64.

Weisman, R. G., Difranco, M. P. (1981). Testing models of delayed sequence discrimination in pigeons: Delay intervals and stimulus durations. Journal of Experimental Psychology: Animal Behavior Processes, 7, 413-424.

Weisman, R. G., Duder, C., \& von Konigslow, R. (1985). Representation and retention of three-event sequences in pigeons. Learning \& Motivation, 16, 239-258.

Weisman, R. G., Gibson, M., \& Rochford, J. (1984). Testing models of delayed sequence discrimination in pigeons: The advance key procedure. Canadian Journal of Psychology, 38, 256-268.

Weisman, R. G., Wasserman, E. A., Dodd, P. W. D., \&arew, M. B. (1980). Representation and retention of two-event sequences in pigeons. Joumal of Experimental Psychology: Animal Behavior Processes, 6, 312-325.

WICKELGREN, W. A. (1972). Trace resistance and the decay of longterm memory. Journal of Mathematical Psychology, 9, 418-455.

Wickelgren, W. A., NormaN, D. A. (1966). Strength models and serial position in short-term recognition memory. Joumal of Mathematical Psychology, 3, 316-347.

YNTEMA, D. B., TRASK, F. P. (1963). Recall as a search process. Journal of Verbal Learning \& Verbal Behavior, 2, 65-74

Zacks, R. T., Hasher, L., Alba, J. W., Sanft, H., \& Rose, K. C. (1984). Is temporal order encoded automatically? Memory \& Cognition, 12, 387-394.

(Manuscript received August 7, 1991; revision accepted for publication June 8,1992 .) 